\title{
Modified iterative sphere decoding algorithm in LTE system
}

\author{
Qin Zhu ${ }^{1}$ Xiao-wen $\mathrm{Li}^{1}$ \\ ${ }^{1}$ Chongqing University of Posts and Telecommunicaiton, Chongqing, China \\ aemail:zhuqin4689@163.com bemail:lixw@cqupt.edu.com
}

Key words: QR sphere decoding iteration algorithm

\begin{abstract}
In the long-term evolution (LTE) system, channel equalization makes compensation to restore the original signal, the paper puts forward iteration sphere decoding algorithm which combines traditional sphere decoding and the improved QR based on the conventional QR decomposition detection algorithm. It can effectively reduce the system complexity. At the same time, in QPSK and 16QAM, the simulation results show that the improved QR iterative sphere decoding algorithm performance is better with higher SNR in AWGN channel.
\end{abstract}

\section{Introduction}

In LTE system, the receiver uses signal detection and estimation methods to retrieve information from the received data to recover the transmitted signals. This process is referred to as channel equalization. This paper expounds the principle of decomposition algorithm and improved QR decomposition iterative detection algorithm. An improved algorithm is presented by combining QR algorithm with sphere decoding methodology. In addition, the improved algorithm will be compared with the Maximum Likelihood (ML). This paper is organized as follows: the QR decomposition detection principles will be described in section 2. Section 3 will present the improved iterative QR decomposition detection method. The combination of sphere decoding methodology and improved QR decomposition detection method will be given in section 4. Section 5 shows the simulation results, conclusions are drawn in section 6 .

\section{QR decomposition detection principle}

Channel model: $\mathrm{Y}=\mathrm{HX}+\mathrm{N} . \mathrm{QR}$ will decompose the channel impact response matrix $\mathrm{H}$ at $(\mathrm{k}, \mathrm{l})$. The result is the unitary matrix $\mathrm{Q}$ and the upper triangular matrix $\mathrm{R}$, and then the matrix $\mathrm{QH}$ left multiplied by the received vector $\mathrm{Y}$, after conversion. The result is received vector $\tilde{y}$. after conversion expressions are as follows:

$$
\tilde{y}=Q^{H} Y=R X+Q^{H} n=R X+\eta
$$

After decomposing, we can obtain the equations:

$$
\begin{aligned}
& \tilde{y}_{n T}=r_{n T, n T} x_{n T}+\eta_{n T} \\
& \left\{\begin{array}{l}
\tilde{y}_{n T-1}=r_{n T-1, n T-1} x_{n T-1}+r_{n T-1, n T} x_{n T}+\eta_{n T-1} \\
\cdots \cdots \cdots \cdots \\
\tilde{y}_{1}=r_{1,1} x_{1}+\sum_{j=2}^{n_{T}} r_{1, j} x_{j}+\eta_{1}
\end{array}\right.
\end{aligned}
$$

For the first equation, it can be processed firstly, get $\hat{x}_{n T}=Q\left[\frac{y_{n T}}{r_{n T, n T}}\right]$ which is the estimated value of $x_{n T}$, mapping $\frac{y_{n T}}{r_{n T, n T}}$ to the nearest constellation point of the distance to eliminate the interference caused by $x_{n T}$ and get $\hat{x}_{n T-1}=Q\left[\frac{\tilde{y}_{n T-1}-r_{n T-1, n T} X_{n T}}{r_{n T-1, n T-1}}\right.$. Repeat the above steps, get the estimated value of all complex-valued symbols. Whereby based on the received signal Y, we can get the detected transmission signal $\mathrm{X}$. 


\section{Improved QR decomposition Iterative cycle detection algorithm}

First of all, apply QR and retain the estimated value of the last detection layer, abandon the other layers of the estimated values. Adjust the channel impulse response matrix of a column vector, so that the final estimation value of the last detection layer. Make signal detection for $\mathrm{H}$ which is adjusted using QR, and retain the last detection layer and adjusted to the next previous layer which is first detected layer, repeat the process until all of the symbols to be detected are completed. The basic steps are as follows:

(1)Use the traditional QR to obtain layers of the detected value $\hat{X}_{n T} 、 \hat{X}_{n T-1} . . \hat{X}_{1}$

(2)Assume $\mathrm{i}=1$;

(3)Adjust the order, and modulate the original detection order to get [M, N], adjust the channel impulse response matrix $H$ according to the adjusted detection order, get $\left[h_{i+1} \cdots h_{n T} h_{1} \cdots h_{i-1} h_{i}\right]$;

(4)Make QR decomposition impact on the adjusted channel response matrix;

(5)Keep the last $\hat{x}_{i+1}$ as detection value;

(6)Assume $\mathrm{i}=\mathrm{i}+1$, if $\mathrm{i}<\mathrm{nT}$, then repeat step 3 , if $\mathrm{i}=\mathrm{nT}$, representing all symbols are detection finished.

\section{Sphere decoding algorithm based on the improved QR iteration of the loop}

Sphere decoding is to solve the following problems: $\min _{s \in D \subset Z^{m}}\|r-H s\|_{2}$. Where $Z^{m}$ is an integer lattice point set, $r$ is the received signal, and $s$ is the transmission signal. Now it is needed to find an $r$ to satisfy $d^{2} \geq\|r-H s\|_{2}^{2}$, where $d$ is the selected appropriate radius. Based on the improved QR decomposition algorithm derivation, concrete steps are as follows:

(1) As known is first input condition of receiver: ${ }^{d}, Q=\left[Q_{1}, Q_{2}\right], \mathrm{R}, \mathrm{x}, \quad y=Q_{1}^{*} d$

(2) QR decomposition on channel matrix $\mathrm{H}$, assume $\mathrm{N} \geq \mathrm{M}$, we have

$H=\left[Q_{1}, Q_{2}\right]\left[\begin{array}{l}R \\ 0_{(N-M)^{*} M}\end{array}\right]$

The decomposition of $\mathrm{H}$ is

$d_{2}^{2}=d_{s}^{2}-\left\|Q_{2}^{H} y\right\|_{2}^{2}, y=Q_{1}^{H} r$

So,it can be decomposed according to the following:

$d^{2} \geq\left\|x-\left[\begin{array}{ll}Q_{1} & Q_{2}\end{array}\right]\left[\begin{array}{l}R \\ 0\end{array}\right] s\right\|^{2}=\left\|\left[\begin{array}{l}Q_{1}^{*} \\ Q_{2}^{*}\end{array}\right] x-\left[\begin{array}{l}R \\ 0\end{array}\right] s\right\|^{2}=\left\|Q_{1}^{*} x-R s\right\|^{2}+\left\|Q_{2}^{*} x\right\|$

Movethe right shift to the left: $d^{2}-\left\|Q_{2}^{*} x\right\| \geq\left\|Q_{1}^{*} x-R s\right\|^{2}$, and Assume: $y=Q_{1}^{*} x$, $d^{\prime 2}=d^{2}-\left\|Q_{2}^{*} x\right\|^{2}$, bring this condition to the above formula will be:

$$
d^{\prime 2} \geq \sum_{i=1}^{m}\left(y_{i}-\sum_{j=1}^{m} r_{i j} s_{j}\right)^{2}
$$

(3) get its expansion inequalities

$$
d^{2} \geq\left(y_{m}-r_{m, m} s_{m}\right)^{2}+\left(y_{m-1}-r_{m-1, m} s_{m}-r_{m-1, m-1} s_{m-1}\right)^{2}+\cdots
$$

after decomposition and Change to the form of interval:

$\left\lceil\frac{-d^{\prime}+y_{m}}{r_{m, m}}\right\rceil \leq s_{m} \leq\left\lfloor\frac{d^{\prime}+y_{m}}{r_{m, m}}\right\rfloor$

(4)After getting $S_{m}$, make $d_{m-1}{ }^{2}=d^{2}-\left(y_{m}-r_{m, m} s_{m}\right)^{2}$ and $y_{m-1 \mid m}=y_{m-1}-r_{m-1, m} s_{m}$, get $d^{\prime 2} \geq\left(y_{m}-r_{m, m} s_{m}\right)^{2}+\left(y_{m-1}-r_{m-1, m} s_{m}-r_{m-1, m-1} s_{m-1}\right)^{2}+\cdots$

As known, the first and second of left formula is necessary condition, it can be launched the value space by the necessary condition 


$$
\left\lceil\frac{-d_{m-1}+y_{m-1 \mid m}}{r_{m-1, m-1}}\right\rceil \leq s_{m} \leq\left\lfloor\frac{d_{m-1}+y_{m-1 \mid m}}{r_{m-1, m-1}}\right\rfloor
$$

And so on, it can pass out the value space of $S_{m-2}, \ldots . . . S_{1}$.

(5)Assume $\mathrm{k}=\mathrm{m}, d_{m}^{2}=d^{2}-\left\|Q_{2}^{*} x\right\|^{2}$.

(6) Set $S_{k}$ of the lower bound is $\left\lceil\frac{d_{k}{ }^{\prime}+y_{k \mid k+1}}{r_{k, k}}\right\rceil-1$ the upper bound is $\left\lfloor\frac{d_{k}{ }^{\prime}+y_{k \mid k+1}}{r_{k, k}}\right\rfloor$.

(7) It begins traverse from the smallest integer $s_{k}$ where $\mathrm{k}=\mathrm{m}$, if $s_{k} \leq U B\left(s_{k}\right)$, then $\mathrm{k}=\mathrm{k}-1, y_{k \mid k-1}=y_{k}+\sum_{j=k+1}^{m} r_{k, j} s_{j}, d_{k}^{\prime 2}=d_{k+1}{ }^{2}-\left(y_{k+1}-r_{k+1, k+1} s_{k+1}\right)^{2}$.In the same way, set ${ }^{2}{ }_{k-1}$ and begin to judge $s_{k-1} \leq U B\left(s_{k-1}\right)$ form the minimum one of $s_{k-1}$, if meet to condition, then $\mathrm{k}-1$ until $\mathrm{k}=1$, cancel $s_{k}, s_{k-1} \ldots \ldots s_{1}$ and save $s$ and the error $d_{m}^{\prime 2}=d_{1}^{\prime 2}+\left(y_{1}-r_{1,1} s_{1}\right)^{2}$.

(8)In step 7,judging $s_{k} \leq U B\left(s_{k}\right)$ after decreasing $\mathrm{k}$, if the conditions are not satisfied, then $k=k+1$,stop operation until $k=m+1$,otherwise, ${ }_{k}=s_{k}+1$, continue to traverse.

(9)The sphere decoding algorithm will traverse all value, if it can not meet the condition ,then cancel it, and compare every kind of error $d_{m}^{\prime 2}=d_{1}^{\prime 2}+\left(y_{1}-r_{1,1} s_{1}\right)^{2}$, save the value of which error is the least.

\section{Simulation Results}

The following four plots show that the result of simulation which using Monte Carlo simulation in the QPSK and 16QAM in $2 * 2$ and $4 * 4$ of MIMO, it is BER of comparison between ML, sphere decoding algorithm ( SD ), improved QR detection algorithm(rev-QR) and proposed iterative sphere decoding algorithm based on the improved QR the algorithm(rev-QR-SD) to show different performance.

Based on the simulation results shown in the figures, we can conclude that: comparison of the figure 1 and figure 2, figure 3 and Figure 4, in low SNR, the above algorithms have the consistent performance, in $2 * 2$ and $4 * 4$ MIMO cases, using 16QAM modulation algorithm, in high SNR, improved sphere decoding have the better performance. With the antenna number increases, the performance of the four algorithms have the growing gap. In high SNR, improved sphere decoding have more obvious advantages.

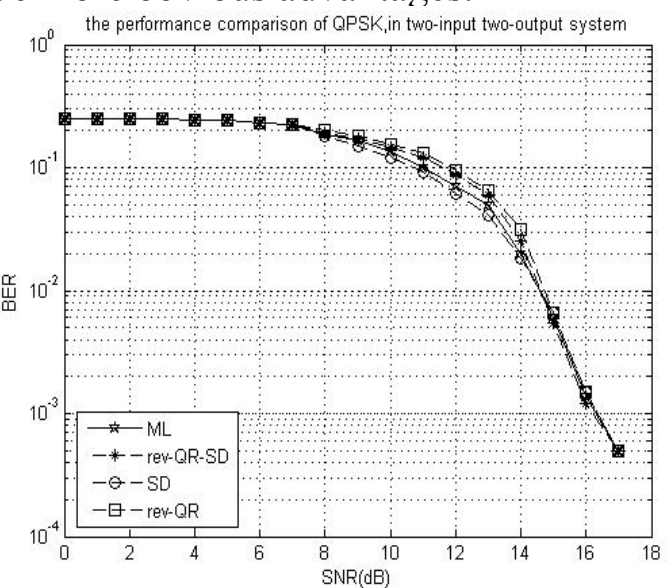

Figure 1 The performance comparison of QPSK, in $2 * 2$ system

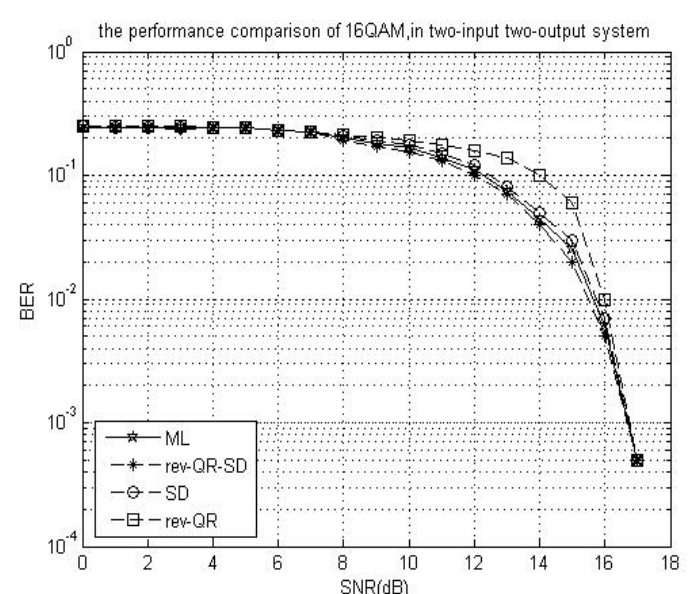

Figure 2 The performance comparison of $16 \mathrm{QAM}$, in $2 * 2$ system 


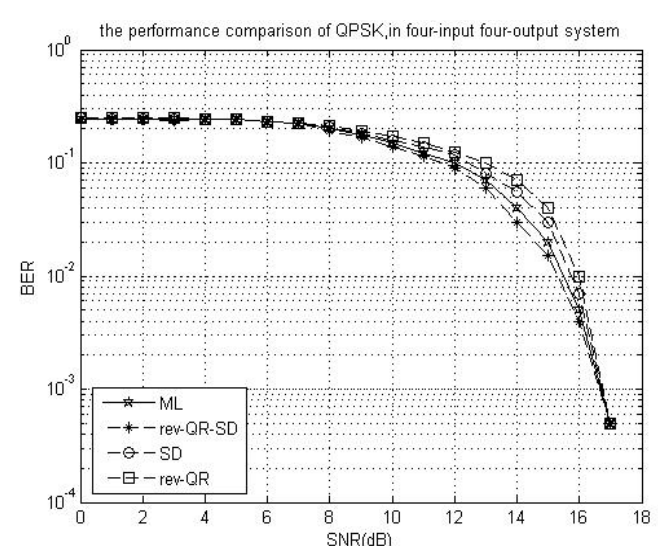

Figure 3 The performance comparison of QPSK, in $4 * 4$ system

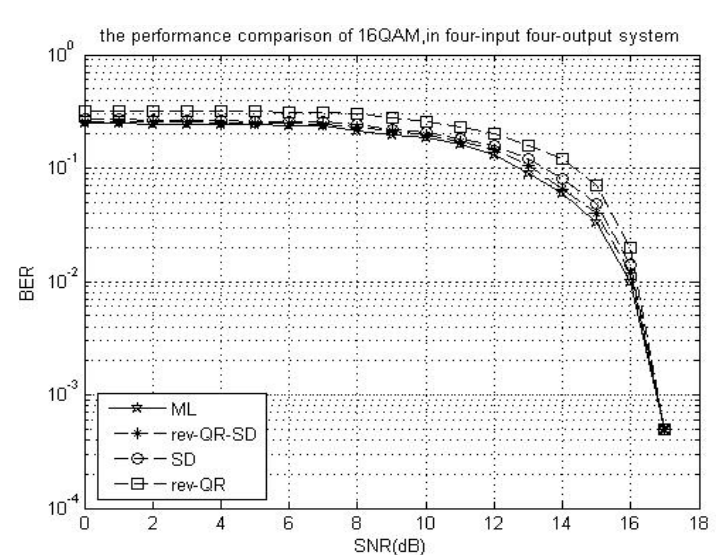

Figure 4 The performance comparison of 16QAM, in $4 * 4$ system

\section{Conclusions}

Based on the traditional QR detection algorithm, this paper proposed an improved iterative QR decomposition detection method. The channel can make full use of the diversity gain of the last detection layer, which will result an improvement of the detection performance. In addition, the improved method was combined with the sphere decoding methodology to further enhance the system performance. Simulation results showed that the proposed QR iterative sphere decoding algorithm can not only significantly reduce the computational complexity, but also improve the system achievability.

\section{References}

[1] Jia Shen,Siqiang Suo,Haiyang Quan and so on. 3GPP long term evolution ( LTE ) technology principle and system design [M].Bei Jing:The people post and Telecommunications Press, 2008.280-315.

[2] Xiaowen LI,Ge Gou,Fatang Chen. an improved signal detection algorithm in LTE system LTE. Application of electronic technology.2010.11:131-134

[3] Bing Gong,Feiqi Deng. based on QR decomposition model iterative detection algorithm in MIMO-OFDM system [J].Application of electronic technology.2009.12:

[4] A new QR-based block least mean squares (QR-BLMS) algorithm for adaptive parameter estimation [J].2003 IEEE International Conference.2003,VI-409-12.

[5] Interpolation-Based QR Decomposition and Channel Estimation Processor for MIMO-OFDM System. IEEE Transactions[J],2011,1129-1141

[6] 3GPP TS 36.101 v9.3.0: User Equipment (UE) radio transmission and reception(Release 9). 2010.3 